\title{
Using guided individualised feedback to review self-reported quality of life in health and its importance
}

\begin{abstract}
This pilot study investigated the effects of providing guided, individualised feedback on subjective quality of life (QoL), using results from the multi-dimensional WHOQOL-BREF profile.
\end{abstract}

Participants ( $\mathrm{n}=129 ; 85$ chronically ill) were recruited in the community, and primary care. They were randomized to receive written or verbal guidance on interpreting a new graphical summary profile, which simultaneously presented (a) their individual self-ratings of QoL, and (b) the importance attributed to each QoL dimension. Before and after feedback, participants completed health status, subjective QoL, QoL importance, goal-oriented QoL, and mood measures.

Receiving individualised feedback was associated with increased psychological QoL, with modest effect size. No effects were found for physical, social or environmental QoL or QoL importance, health status, mood or goal-oriented QoL. There were no differences between modes of delivering guidance, indicating equal effectiveness. Chronic illness participants reported poorer QoL, moved more slowly towards their QoL goals, and had larger differences between core QoL and QoL Importance than healthy participants.

Guided individualised empirical feedback about QoL judgements could be used to promote psychological wellbeing. Although professional interpretation of feedback is unnecessary, if shared, patients' profiled WHOQOL information could support selfmonitoring, self-management, and clinical decision-making.

Keywords: quality of life, WHOQOL-BREF, health, feedback, wellbeing 


\section{Introduction}

In 2011, the UK Government launched a new mental health strategy: 'No Health Without Mental Health' (Department of Health), reflecting increasing policy focus on the importance of wellbeing. Recent healthcare models have also placed great emphasis on supporting active participation in personal care. Consequently there has been rapid growth in using patientreported outcome measures (PROMs) in the NHS, like health-related quality of life (QoL) (NHS England, 2013). While PROMs mostly serve to audit the outcomes of healthcare, they can also assist health professionals by providing empirical evidence of intervention outcomes on wellbeing, although these are rarely observed or formally assessed (Valderas et al., 2008).

At an individual level, PROMs have particular value in enabling patients and their health professionals to jointly identify goals and priorities for future health and health care (Marshall, Haywood, \& Fitzpatrick, 2006), and the NHS Operating Framework states the organisation should '...put patients at the centre of decision-making, with their experience of health' (Department of Health, 2011, p. 8). International QoL research has increasingly focused on person-centred methods (Hahn, 2012; Iris, Debacker, Benner, Hammerman, \& Ridings, 2012; Skevington \& McCrate, 2012), underscoring current shifts towards targeting treatments in personalised healthcare. This approach requires new ways to investigate the QoL of individuals, in contrast to epidemiological research.

Although person-centred studies have examined the effects of QoL assessment on individual patient satisfaction (De Wit et al., 2008; Gutteling et al., 2008; Hilarius, Kloeg, Gundy, \& Aaronson, 2008; Mills, Murray, Johnston, Cardwell, \& Donnelly, 2009; Rosenbloom, Victorson, Hahn, Peterman, \& Cella, 2007), few have specifically examined whether patients' QoL can improve as an consequence of completing a QoL measure and receiving feedback about it. This represents an intervention in its own right, and evidence for its efficacy is contradictory. While some studies found no effects (Hilarius et al., 2008; 
Rosenbloom et al., 2007; Santana et al., 2010), others provide limited evidence of positive outcomes such as improved emotional wellbeing (Velikova et al., 2004), disease-specific QoL and mental QoL (Gutteling et al., 2008), behavior, psychosocial health and family activities (De Wit et al., 2008). Although indicating that QoL feedback is an important mechanism whereby wellbeing can be influenced, the exact process remains unclear, as feedback methods vary widely.

Recently, there has been renewed interest in measuring the importance that individuals attach to different aspects of their QoL (Baile, Palmer, Bruera, \& Parker, 2011; Snyder et al., 2011), as distinct from importance to populations (da Rocha, 2011; Molzahn, Kalfoss, Skevington, \& Schick Makaroff, 2011; Molzahn, Skevington, Kalfoss, \& Schick Makaroff, 2010; Skevington \& O'Connell, 2004; Wu, 2009). Taking an unusual approach, Skevington and O'Connell (2004) examined international data about QoL and its importance. They found that the poorest QoL could be identified where a particular dimension of QoL was rated not only as poor (low scores), but also as very important (high scores) and that where there was a large difference (gap) between the two ratings, high importance had a supplementary disenhancing effect on poor QoL. They suggested that this knowledge could help patients and professionals target areas of wellbeing for improvement.

The main aim of the present study was to pilot a new procedure to give graphical feedback on both types of information, and investigate its effect on wellbeing. This is an original investigation in feedback research. We predicted that self-generated empirical information would promote self-awareness of current states, and movement towards future personal QoL goals. Self-management interventions based on this premise affect wellbeing positively during chronic illness (Ditewig, Blok, Havers, \& van Veenendaal, 2010; Labrecque et al., 2011) and the feasibility of technology -based symptom monitoring has been recently demonstrated in cancer patients (Yount et al., 2013). It was, therefore, 
important to apply this pilot research in a chronic illness population, and healthy participants were recruited for comparison purposes.

The investigation was grounded in Carver \& Scheier's self-regulation theory (SRT) (Carver \& Scheier, 1982, 1998) which asserts that the function of self-regulation is to reduce sensed discrepancies between an individual's judgement of their actual state and their desired state. To do this, individuals use information from feedback to adapt to, or steer towards their goal(s). Perceived progress, or lack of it, is theorised to have affective and behavioural consequences. This is relevant to our study because achieving one's goals is also theorised to be an important source of information that people use to judge their QoL. The present study is underpinned by the World Health Organization (WHO) definition of QoL: 'An individuals' perceptions of their position in life, in the context of the culture and value systems in which they live, and in relation to their goals, expectations, standards and concerns.' (The WHOQOL Group, 1995, p. 1405). In view of the synergy between selfregulation mechanisms and QoL feedback, we sought to measure goal-related QoL.

Based on previous findings on the positive effects of QoL feedback (De Wit et al., 2008; Velikova et al., 2004), we hypothesised that inspecting and interpreting individualised QoL feedback after completing these measures would promote general wellbeing. In the absence of suitable prior research, we could not predict whether chronic illness and healthy participants would differ under these conditions, and if so how. However, we expected that chronic illness participants would report poorer physical QoL than healthy (Dickson, Toft, \& O'Carroll, 2009; Kilian, Matschinger, \& Angermeyer, 2001).

We investigated whether verbal guidance on how to interpret the QoL results delivered at the time of feedback would be more strongly associated with improvements in wellbeing than following written guidance from printed instructions. Patients are keen to discuss their QoL results in clinical settings following verbal guidance (Detmar, Aaronson, Wever, Muller, 
\& Schornagel, 2000), but little comparative work with written feedback has been conclusive so we had no predictions about modes of administering feedback. As prior research identifies the poorest QoL (Skevington \& O'Connell, 2004), we predicted that people with chronic illness would have poorer QoL on some dimensions of the profile, and would rate these aspects of QoL as more important than healthy participants, so resulting in greater differences between core QoL and importance. Furthermore, this is the first study to use a new shortform WHOQOL Importance measure compatible with facets of WHOQOL-BREF.

\section{Methods}

\section{Participants}

A UK sample was purposively recruited in the local community and primary care. We designed a pilot study for a feedback intervention where participants acted as their own individual controls, and comparisons were made between chronic illness and well subgroups. Ethical approval for the study was granted by the University of Bath Psychology Department Research Ethics Committee, and the South West 5 NHS Research Ethics Committee. The sample was designed to target $70 \%$ participants with a chronic illness, and $30 \%$ healthy, for comparison. Pragmatically, it is important for health and social care services to know whether this intervention is effective in limiting long-term conditions. The illness subgroup was screened for length of diagnosis, to ensure their condition was chronic. They were included if diagnosed with a chronic physical illness 12 or more months previously. Co-morbidities were recorded and participants were asked which chronic condition most affected their QoL.

Advertisements inviting participation from healthy and chronically ill people were installed on UK websites of the Multiple Sclerosis (MS) Society; Asthma UK; the National Eczema Society; Epilepsy Action and the British Lung Foundation, and n=104 responded, of which $87(84 \%)$ participated. Supplementary recruitment of mostly healthy participants was conducted on a university campus where 33 responded and 17 (52\%) participated. 
Recruitment was also conducted in an NHS Primary Care practice where 250 patients were invited to participate by their General Practitioner. Quota sampling was used to achieve equipoise in the sample design, across a range of chronic physical health conditions. Replies were received from 70 (28\%), and 61 (87\%) participated.

\section{Measures}

Quality of Life and its Importance (The WHOQOL-BREF)

The WHOQOL-BREF is a multidimensional international instrument that assesses subjective QoL over the past two weeks. It contains 26 items covering 25 QoL dimensions; 24 items are scored in one of four domains: physical, psychological, social relationships and environmental QoL. Two general items assess overall health and QoL. Item response is on a five- point Likert interval scale. The WHOQOL-BREF shows good cross-cultural psychometric properties, including in the UK(Skevington \& McCrate, 2012). It is reliable, valid and sensitive to changes in clinical condition (Skevington, Lotfy, \& O'Connell, 2004). It is widely used in clinical trials, for monitoring interventions, and in various service settings. Its patient-centred design and psychometric properties make it suited to individual assessment (Diehr et al., 2006; O'Carroll, Smith, Couston, Cossar, \& Hayes, 2000; Saxena, Carlson, Billington, \& Orley, 2001; Skevington et al., 2004; Skevington \& McCrate, 2012; Skevington \& O'Connell, 2004; The WHOQOL Group, 1998).

Importance items corresponding to topics of QoL facets in the core measure were developed concurrently with the WHOQOL-100 (Saxena et al., 2001); these two measures have very weak correlations in very large datasets (Skevington \& O'Connell, 2004). The Importance measure shows good internal consistency reliability, stability, and has been tested for some aspects of validity (Molzahn et al., 2011; Molzahn et al., 2010; Skevington \& O'Connell, 2004; $\mathrm{Wu}, 2009)$. We used a short form of the WHOQOL-100 Importance scale based on work by Skevington \& O’Connell (2004), where each WHOQOL Importance item 
was selected to match one of the 26 topics in the WHOQOL-BREF.

In the present study, scores from the WHOQOL-BREF and the short WHOQOLImportance measure were used to produce individualised graphical summary profiles (See Figure 1). These profiled results displayed the participant's core QoL responses in bar charts, overlaid with indicators of their importance ratings (red triangles) for every QoL facet. This graphical profile was based on an early version of a CD-ROM based system, developed at $[\mathrm{X}]$, and used by patients and physicians to inspect the results in a previous pilot study.

\section{[INSERT FIGURE 1 HERE]}

\section{Health Status (EQ-5D)}

Health status overall was measured using the visual analogue scale of the EQ-5D (The EuroQoL Group, 2010) $(0=$ worst imaginable health; $100=$ best imaginable health). The National Institute for Clinical Excellence (NICE) recommends the EQ-5D as the preferred UK measure of health-related QoL in adults (National Institute for Health and Clinical Excellence, 2008), and its validity in assessing chronic illnesses is confirmed (Dyer, Goldsmith, Sharples, \& Buxton, 2010; Janssen, Lubetkin, Sekhobo, \& Pickard, 2011; Pickard et al., 2008).

\section{Mood (PANAS)}

The Positive and Negative Affect Schedule (PANAS) (Watson, Clark, \& Tellegen, 1988) was selected to measure positive and negative affect independently. This instrument contains two internally consistent 10-item mood scales that are largely uncorrelated and stable (Watson et al., 1988). Items are rated from 1 to 5, and scores summed for each affect type (range 10-50). The PANAS shows good psychometric properties in general, clinical and non-clinical UK populations (Crawford \& Henry, 2004; Ostir, Smith, Smith, \& Ottenbacher, 2005). Goal-Oriented Quality of Life (GOSS)

The goal-oriented subjective status scale (GOSS) Dibb and Yardley (2006), measures 
perceptions of how quickly people believe they are moving towards, or away from five specific goals relating to: family/relationships, health, work/finances, social life/activities, spirituality and beliefs. The GOSS has good internal consistency (alpha $\geq 0.8$ ) and is meaningfully related to the 'change in health' item of another health status measure - the SF36 (Jenkinson, Wright, \& Coulter, 1993). For this study, the wording of the GOSS was adapted so as to map it conceptually onto the six QoL domains of the WHOQOL-100 (The WHOQOL Group, 1995): physical health, psychological, social relationships, environment, independence and spiritual QoL. Each of the six items in this revised measure (the GOSS-Q) asks about perceived movement 'today' in relation to ideal QoL $(1=$ "moving very quickly away"; 7 = "moving very quickly towards" (range from $7-42$ ). The adapted measure has good internal consistency reliability (alpha $\geq 0.8$ ) (Llewellyn \& Skevington, 2014).

\section{Procedure}

Having given fully informed consent, participants completed all baseline (T1) measures at home, online or on paper, and returned their questionnaires to the primary researcher (AL). Participants were stratified by health status (illness or health), then randomly allocated to one of two conditions - verbal or written guidance - using independent computer generated randomization (www.randomizer.org). Individualised graphical summary profiles from the WHOQOL-BREF and the short WHOQOL-Importance measures were printed, in colour, to provide the mechanism for subsequent feedback according to experimental condition.

In the verbal guidance condition, feedback session appointments were made by telephone and individual graphical summary profiles were sent by post or email to the participants in time for these appointments (within 1-3 weeks of baseline measurement, depending on participants' availability). During the appointment, participants reviewed and interpreted their graphical summary profile while receiving guidance on how to understand the profile from the researcher (AL) over the telephone, using a prepared script. In the written 
guidance condition, the graphical summary profile was sent to participants within one week of baseline measurement, together with printed instructions providing directions about how to review and interpret the profile by following a series of steps. The design of the graphical summary profile was identical in both conditions and instruction protocols were matched in terms of the steps participants should follow when reviewing and interpreting their graphs. Any interaction with participants concerning their scores was restricted to factual and descriptive comments; the researcher was careful not to impute meanings. The methodology of the two conditions had been piloted and tested previously for feasibility and acceptability (Llewellyn, 2012).

In both feedback conditions, participants were first directed to look at all of his/her QoL core scores, and to identify the facets where QoL was rated as good (>3.0). Then they examined Importance scores to find any large differences between Importance and core QoL. In particular they focused on facets where scores indicated poor QoL $(<3.0)$ and where that facet was also considered to be very or extremely important ( $>3)$, implementing procedures based on Skevington and O'Connell's (2004) findings. It was pointed out to participants that these were the areas where they might want to consider what they could do to make the gap smaller. For ethical reasons, the final instruction was for them to attend to facets where they had rated both QoL and Importance as high, and so were positive.

Participants were asked to complete the time 2 (T2) measures immediately after reviewing the graphical summary profile, but without referring back to it. We thereby intended that the T2 measures would capture their new post-feedback self-judgements, not ones they might have derived through systematic comparisons with previous answers.

\section{Data analysis}

The WHOQOL-BREF scores were transformed so that the four domains (physical health, psychological, social relationships and environment) were scored from 0-100, using a 
WHOQOL Group SPSS syntax file (obtainable from:

http://apps.who.int/iris/bitstream/10665/77932/1/WHO_HIS_HSI_Rev.2012.03_eng.pdf).A new syntax was written by the authors to similarly transform the WHOQOL Importance scores into Importance domains. SPSS v18 was used for statistical analysis. Normality testing showed that skewness and kurtosis were acceptable for most variables. Negative Affect scores (PANAS) were reciprocally transformed (Hair, Anderson, Tatham, \& Blacke, 1999).

Factorial repeated measures MANOVA was used to examine differences over time, by health status (ill or healthy), and by experimental condition (verbal or written guidance). In the first analysis we included five dependent variables (DVs): each of the four WHOQOLBREF core domains and the EQ-5D VAS rating. All were acceptably correlated. MANOVA was applied to the WHOQOL Importance domain scores, which were poorly correlated with the WHOQOL-BREF. Mixed-factorial ANOVAs examined differences over time and between groups for the other DVs: GOSS-Q and PANAS. Bonferroni corrections and Greenhouse-Geisser adjustments factors were applied (Dancey \& Reidy, 2004). Effect size was calculated using Cohen's guidelines for interpreting $F$ (Cohen, 1988), with equivalent values of partial eta squared, as generated by SPSS (Kinnear \& Gray, 2010). We investigated the differences between core QoL and Importance scores for each health status group at each time point, using dependent $t$-tests, with Bonferroni corrections. Significance was set at $p=$ 0.013 to correct for multiple testing.

\section{Results}

Baseline questionnaires (T1) were completed by 165 participants, 129 of whom also completed T2. Seventy-four $\%$ of those with chronic illness completed both sets of questionnaires, compared to $87 \%$ of healthy people, suggesting physical health may have been a factor in attrition. Of those completing questionnaires twice, $66 \%$ were women; $35 \%$ were aged $18-44$ years, $52 \% 45-74$ years and 10\% 75+. Attrition rates were similar for men 
$(25 \%)$ and women $(20 \%)$, and varied little by age band. After randomization, 71 participants (55\%) received verbal guidance, of whom $65 \%$ reported a chronic illness; and $58(45 \%)$ received written guidance $(67 \%$ ill $)$.

Most participants (64\%) had received tertiary education (college, university or postgraduate), were White (96\%), and lived with a spouse, partner or family (76\%). Most resided in sub/urban areas (69\%) and 28\% were retired, 34\% were employed full-time, and $12 \%$ part-time. The chronic illness group $(n=85)$ was a heterogeneous sample of people reporting chronic illnesses (defined by the International Classification of Diseases (World Health Organization, 2010)). These included musculoskeletal and connective tissue (21\%); respiratory system (19\%); nervous system (19\%); circulation (13\%); dermatology (11\%); and endocrine, nutrition and metabolic conditions (8\%); others $9 \%$.

\section{Outcome Changes between Pre-test (T1) and Post-test (T2)}

The MANOVA analysis of the WHOQOL-BREF and EQ-5D VAS showed a significant main effect, with scores increasing over time $\left(p<.05, \eta^{2}=.10\right)$ (see Table 1). Examination of contrasts revealed that although mean scores were mostly higher at T2, only the WHOQOLBREF psychological domain was significant $\left(p<.01, \eta^{2}=.06\right)$, indicating that the main effect was associated with change in this domain. These findings support our hypothesis that being guided through individualised QoL feedback would bring about improvements in wellbeing.

The EQ-5D VAS and WHOQOL-BREF domain scores distinguished between ill and healthy groups $\left(p<.001, \eta^{2}=.21\right)$, showing a large effect size. As expected, participants reporting chronic illness rated their physical health as poorer on both measures than healthy people (EQ-5D VAS: $p<.001, \eta^{2}=.17$, WHOQOL-BREF physical domain: $p<.001, \eta^{2}=$ .16). No significant differences were found between verbal and written guidance groups on any variable. Furthermore there were no significant interactions between time, health status and experimental condition. 
[INSERT TABLE 1 HERE]

The PANAS subscales showed no significant differences between T1 and T2 and effects of health status or experimental condition, or any significant interactions. Therefore our findings did not support earlier predictions of improvement in affect. GOSS-Q scores did not change significantly over time, however there was a significant between-subjects effect in health status, with medium effect size $\left(F(1,125)=15.16, p<.001, \eta^{2}=.11\right)$. Goal-oriented QoL scores were significantly lower for the ill group than for healthy participants. Although the overall means indicated that both groups were moving towards, rather than away from their ideal QoL, higher scores for healthy participants indicated more rapid movement in this positive direction. There were no significant interactions between time, health status and experimental condition.

MANOVA showed that WHOQOL Importance domain scores did not change significantly over time. However, contrary to our hypothesis, healthy participants rated the importance of QoL more highly than ill participants $\left(p<.01, \eta^{2}=0.11\right)$. Contrasts revealed that this between-groups difference was related to WHOQOL Importance scores in both the physical and psychological domains $\left(d f=1,122\right.$, Importance physical: $p<.001, \eta^{2}=.09$; Importance psychological: $\left.p<.05, \eta^{2}=.04\right)($ See Table 2).

[INSERT TABLE 2 HERE]

\section{Differences between Quality of Life and the Importance of Quality of Life}

A number of significant differences were found between WHOQOL core domain scores and respective Importance ratings of ill participants at T1 and T2. Table 2 shows that core physical and psychological QoL scores were rated lower than Importance $(p<.001)$ on both occasions. This effect was found for the environment domain at T1 $(p<.01)$, but not for the social domain. Healthy group means showed a similar pattern, but only psychological core and Importance scores differed at T1 and T2 $(p<.001)$. These findings support the 
prediction of greater differences between core QoL and Importance for those with poorer QoL living with chronic illness.

\section{Discussion}

The aim of this study was to explore whether a novel approach to receiving feedback on subjective judgements would lead to changes in how people consider their QoL, their orientation towards their QoL goals, and their mood. Within this design, we also examined whether the method by which feedback was administered had different effects. Our findings confirm that receiving feedback on subjective QoL and importance judgements was be positive for the psychological domain, suggesting improved mental health although the size of this effect was modest. This result supports the prediction that sharing QoL results with individuals who have provided the information leads to improved self-judgements. It shows the advantages of a highly patient-centred process, in line with current NHS health care policy. Furthermore these findings demonstrate that the WHQOL-BREF scores are sensitive in detecting change in a community sample. This information has not been widely available.

There was no significant change in the three other WHOQOL-BREF domains or health status on the EQ-5DVAS. These results are consistent with reports by Velikova et al. (2004) and Del Wit et al. (2008) who showed that that emotional wellbeing and mental health improved after QoL feedback but physical QoL showed no change. It therefore seems plausible that physical health indicators like pain, medication, energy, sleep, work, mobility, and daily activities, are more receptive to physical or pharmacological interventions, than to increased self-awareness via a psychological feedback intervention. They may also possibly have greater intrinsic strength than emotional wellbeing, so are more resistant to change. Similarly, no intervention effects were found on core variables of social or environment QoL domains, or on QoL Importance variables, indicating that feedback alone is insufficient to bring about changes in self-judgements in these QoL areas. Combining our approach with 
other psychological interventions that would benefit from structured self-regulation and selfmonitoring could promote mutually enhancing effects. Secondary components relating to behaviour change, such as goal setting, or motivational interviewing, could be usefully developed and applied alongside QoL feedback.

While we had predicted post-feedback changes in mood and goal-related QoL, these were not detected. However, this is consistent with previous studies investigating the effects of QoL assessments with outcome measures other than the WHOQOL-BREF, for example the Functional Assessment of Cancer Therapy (FACT), Palliative Care Quality of Life Index (PQLI) and the Functional Living Index (FLIC) (Hilarius et al., 2008; Mills et al., 2009; Rosenbloom et al., 2007). In the present study it is possible that there were changes but these were not detected by the measures we used. Whether this is due to subtle differences in concepts investigated, or measurement construction is worthy of further investigation. Furthermore, most measures were designed for cross sectional rather than longitudinal research, and for group rather than individual use. It is also plausible that not using diseasespecific measures may have made a difference to our ill sample, as these are generally found to be more responsive to change (Santana et al., 2010), but this would neither have been practicable, nor enabled us to test the generic principle of the impact of QoL feedback.

The present study examined whether providing written or verbal guidance would differentially impact on QoL feedback. We found that both feedback modes worked equally well. As neither showed a superior effect on wellbeing, this has implications for the future implementation of QoL feedback interventions. As a self-management tool, providing written instructions to assist feedback review would be less costly than time spent by a health professional or facilitator providing verbal guidance. The consistency of the same script would improve the reliability of the intervention. It would also allow individuals to review their own feedback in a much wider variety of settings than if a clinical assistant was needed. 
We confirmed an overall QoL difference between those with chronic illness and healthy, but found no difference in psychological QoL. This finding contrasts with previous studies where both physical and psychological QoL of those with chronic illness was poorer than healthy individuals (Dickson et al., 2009; Ovayolu, Ovayolu, \& Karadag, 2011; Salaffi, Carotti, Gasparini, Intorcia, \& Grassi, 2009; Slatkowsky-Christensen, Mowinckel, Loge, \& Kvien, 2007). However, the ill sample in the present study comprised a wide variety of health conditions. Arnold et al. (2004) found that although patients with back problems, rheumatoid arthritis and heart problems reported poorer physical QoL than healthy, patients with lung disorders and migraine differed in psychological functioning. Illness type therefore adds complexity to these group differences in different domains. Future studies comparing QoL in different chronic illness types may enhance our current understanding.

We found participants reported overall movement towards their ideal QoL. However, this movement was perceived to be faster for healthy people, so it is plausible that those with chronic illness may see positive change as more difficult than healthy, or that they invest their energy in coping with the ramifications of their illness, rather than making plans to achieve their QoL goals. This mechanism is in keeping with Schwartz and Drotar's (2009) study where health status was found to affect the pursuit of personal goals during chronic illness in young adults. The present study also showed that physical and psychological QoL were more important for those who were healthy than ill, possibly indicating that chronic illness accompanies lower expectations of QoL in these domains. Although tentative, this interpretation could be fruitfully investigated further.

The results also revealed that the differences between core QoL and Importance scores were larger for those with a chronic illness and were more prevalent across domains. This indicates that those living with chronic illness are more often distant from the QoL goals that they consider to be important, than well people. Furthermore, as other results we obtained 
showed, those with chronic illness are also slower in moving towards their QoL goals than the healthy group. Theoretically this is in keeping with the WHOQOL Group's definition of QoL which indicates that those with the poorest QoL are least likely to meet their '...goals, expectations, standards and concerns' (Skevington \& O'Connell, 2004) and has important implications for clinical practice, where health professionals may need to revise their expectations of what someone with chronic illness can do.

A limitation of the study was the absence of a control group against which to compare those receiving QoL feedback. Including a condition where participants completed questionnaires twice, but did not receive feedback, would have strengthened the study rigor. Similarly, convenience sampling meant we were unable to purposefully match the demographic profiles of the ill and healthy groups in this pilot study and future research with a larger sample would provide a more conclusive understanding. It is also possible that the relatively short duration of the assessment period may have attenuated the potential feedback effect. Allowing longer for feedback to be cognitively and affectively processed may have had greater effect. To our knowledge there is no test of an optimum period for consideration of feedback, and further research would be valuable.

The present study used two different modes of data collection: completing the questionnaires online or using paper and pencil. Paper versions have been employed in the majority of prior studies involving the WHOQOL-BREF, although recently computer-based formats have been reported (Baumann, Ionescu, \& Chau, 2011; Fellinger et al., 2005; Mason, Skevington, \& Osborn, 2004; Skevington \& Day, unpublished). Arguably, having two data collection formats in this study may have introduced an additional variable, however, Chen et al. (2009) found no significant differences between domain scores collected either electronically or using paper and pencil. Paper and touch-screen versions of the EQ-5D VAS also have equivalent properties (Ramachandran, Lundy, \& Coons, 2008) and together these 
results suggest that administration mode does not make an important difference to the way people report their subjective QoL using questionnaires.

At a qualitative level, several participants commented that thinking about QoL was a novel experience. French and Sutton (2011) found that when people were asked to complete questions about issues they had not previously considered, they can provide responses that are generated on the spot, on the basis of normative understandings, rather than on genuine personal reflections. We have no additional information about whether this process was active in our study, but this interpretation has implications for the validity of PROMs measures in general, and for QoL assessment in particular, especially where QoL issues are novel to participants. However, in the present study, all our participants consented in advance to think about their QoL, and therefore had a pretest period to reflect on their QoL before assessment. Providing additional comprehensive information about wellbeing dimensions before recruitment may have made the task less novel but could have conversely served to shape the answers. It is also plausible that self-selection recruitment bias meant those who opted to participate already had an interest in QoL, and may have been considerably selfaware before reviewing their feedback. As such their self-assessments would have been less likely to change after feedback. Similar conclusions have been reached in behaviour change studies, where the effects of planning interventions have been attenuated by the prior plans that participants had already made before taking part in the research (Sniehotta, 2009).

Although we collected data from healthy individuals and a heterogeneous chronic illness sample, small sample sizes precluded analysis of subgroups by illness type. Future studies should investigate the effects of feedback for individuals with different types of chronic illnessess who could be differentially affected by pain, disability, mobility and specific symptoms (Arnold et al., 2004).

The findings indicate that receiving feedback on subjective judgements is associated 
with changes in the way people consider their subjective QoL, and in particular with significant, but small, improvements in psychological wellbeing. This could be an important precursor in making behavioural changes to further improve wellbeing, and ensure longevity of effect. Previous findings suggest that without additional behavioural efforts, these beneficial psychological effects diminish over time (De Wit et al., 2010). However, it may be that psychological QoL change is the necessary foundation upon which changes in physical, social and environmental QoL could subsequently be built. Future studies over longer timescales will be helpful in testing this proposition.

The authors are very grateful to Dr Sanjeev Popli and Mrs Tracy Harris at [xxxxx] Primary Care Trust; the Multiple Sclerosis (MS) Society; Asthma UK; the National Eczema Society; Epilepsy Action, and the British Lung Foundation for publicising this study.

\section{References}

Arnold, R., Ranchor, A. V., Sanderman, R., Kempen, G. I. J. M., Ormel, J., \& Suurmeijer, T. P. B. M. (2004). The relative contribution of domains of quality of life to overall quality of life for different chronic diseases. Quality of Life Research, 13(5), 883-896.

Baile, W. F., Palmer, J. L., Bruera, E., \& Parker, P. A. (2011). Assessment of palliative care cancer patients' most important concerns. Supportive Care in Cancer, 19(4), 475-481.

Baumann, M., Ionescu, I., \& Chau, N. (2011). Psychological quality of life and its association with academic employability skills among newly-registered students from three European faculties. Bmc Psychiatry, 11(63).

Carver, C. S., \& Scheier, M. F. (1982). Control theory - a useful conceptual framework for personality-social, clinical and health psychology. Psychological Bulletin, 92(1), 111135. 
Carver, C. S., \& Scheier, M. F. (1998). On the self-regulation of behaviour. Cambridge, UK: Cambridge University Press.

Chen, W. C., Wang, J. D., Hwang, J. S., Chen, C. C., Wu, C. H., \& Yao, G. (2009). Can the web-form WHOQOL-BREF be an alternative to the paper-form? Social Indicators Research, 94(1), 97-114.

Cohen, J. (1988). Statistical power analysis for the behavioural sciences (2nd ed.). Mahwah, New Jersey, USA: Lawrence Erlbaum Associates.

Crawford, J. R., \& Henry, J. D. (2004). The positive and negative affect schedule (PANAS): Construct validity, measurement properties and normative data in a large non-clinical sample. British Journal of Clinical Psychology, 43, 245-265.

da Rocha, N. (2011). Evaluation of quality of life and importance given to spirituality/religiousness/personal beliefs (SRPB) in adults with and without chronic health conditions. Revista de psiquiatria clínica, 38(1), 19-23.

Dancey, C. P., \& Reidy, J. (2004). Statistics without maths for psychology: using SPSS for Windows (3 ed.). Harlow, UK: Prentice Hall.

De Wit, M., De Waal, H., Bokma, J. A., Haasnoot, K., Houdijk, M. C., Gemke, R. J., \& Snoek, F. J. (2008). Monitoring and discussing health-related quality of life in adolescents with type 1 diabetes improve psychosocial well-being - A randomized controlled trial. Diabetes Care, 31(8), 1521-1526.

De Wit, M., De Waal, H., Bokma, J. A., Haasnoot, K., Houdijk, M. C., Gemke, R. J., \& Snoek, F. J. (2010). Follow-up results on monitoring and discussing health-related quality of life in adolescent diabetes care: benefits do not sustain in routine practice. Pediatric Diabetes, 11(3), 175-181.

Department of Health. No health without mental health: a cross-government mental health outcomes strategy for people of all ages. Retrieved 6th June 2011, from 
http://www.dh.gov.uk/en/Publicationsandstatistics/Publications/PublicationsPolicyAn dGuidance/DH_123766

Department of Health. (2011). The Operating Framework for the NHS in England 2012/2013. Retrieved 4th January, 2013, from https://http://www.gov.uk/government/uploads/system/uploads/attachment_data/file/2 16590/dh_131428.pdf

Detmar, S. B., Aaronson, N. K., Wever, L. D. V., Muller, M., \& Schornagel, J. H. (2000). How are you feeling? Who wants to know? Patients' and oncologists' preferences for discussing health-related quality-of-life issues. Journal of Clinical Oncology, 18(18), $3295-3301$.

Dibb, B., \& Yardley, L. (2006). How does social comparison within a self-help group influence adjustment to chronic illness? A longitudinal study. Social Science \& Medicine, 63(6), 1602-1613.

Dickson, A., Toft, A., \& O'Carroll, R. E. (2009). Neuropsychological functioning, illness perception, mood and quality of life in chronic fatigue syndrome, autoimmune thyroid disease and healthy participants. Psychological medicine, 39(9), 1567-1576.

Diehr, P., Derleth, A., McKenna, S., Martin, M., Bushnell, D., Simon, G., \& Patrick, D. (2006). Synchrony of change in depressive symptoms, health status, and quality of life in persons with clinical depression. Health and Quality of Life Outcomes, 4(27).

Ditewig, J. B., Blok, H., Havers, J., \& van Veenendaal, H. (2010). Effectiveness of selfmanagement interventions on mortality, hospital readmissions, chronic heart failure hospitalization rate and quality of life in patients with chronic heart failure: A systematic review. Patient Education and Counseling, 78(3), 297-315. 
Dyer, M. T., Goldsmith, K. A., Sharples, L. S., \& Buxton, M. J. (2010). A review of health utilities using the EQ-5D in studies of cardiovascular disease. Health Qual Life Outcomes, 8(13), 1-12.

Fellinger, J., Holzinger, D., Dobner, U., Gerich, J., Lehner, R., Lenz, G., \& Goldberg, D. (2005). An innovative and reliable way of measuring health-related quality of life and mental distress in the deaf community. Social Psychiatry and Psychiatric Epidemiology, 40(3), 245-250.

French, D. P., \& Sutton, S. (2011). Does measuring people change them? The Psychologist, 24(4), 272-274.

Gutteling, J. J., Darlington, A.-S. E., Janssen, H. L. A., Duivenvoorden, H. J., Busschbach, J. J. V., \& Man, R. A. (2008). Effectiveness of health-related quality-of-life measurement in clinical practice: a prospective, randomized controlled trial in patients with chronic liver disease and their physicians. Quality of Life Research, 17, 195-205.

Hahn, J. E. (2012). Minimizing Health Risks Among Older Adults With Intellectual and/or Developmental Disabilities: Clinical Considerations to Promote Quality of Life. Journal of gerontological nursing, 38(6), 11-17.

Hair, J. F., Anderson, R. E., Tatham, R. L., \& Blacke, W. C. (1999). Multivariate Data Analysis (5th ed.). Englewood Cliffs, N.J.: Prentice-Hall.

Hilarius, D. L., Kloeg, P. H., Gundy, C. M., \& Aaronson, N. K. (2008). Use of health-related quality-of-life assessments in daily clinical oncology nursing practice. Cancer, 113(3), 628-637.

Iris, M., Debacker, N. A., Benner, R., Hammerman, J., \& Ridings, J. (2012). Creating a quality of life assessment measure for residents in long term care. Journal of the American Medical Directors Association, 13(5), 438-447. 
Janssen, M. F., Lubetkin, E. I., Sekhobo, J. P., \& Pickard, A. S. (2011). The use of the EQ5D preference-based health status measure in adults with Type 2 diabetes mellitus. Diabetic Medicine, 28(4), 395-413.

Jenkinson, C., Wright, L., \& Coulter, A. (1993). Quality of life measurement in health care: a review of measures and population norms for the UK SF-36. Oxford, UK: Health Service Research Unit.

Kilian, R., Matschinger, H., \& Angermeyer, M. C. (2001). The impact of chronic illness on subjective quality of life: a comparison between general population and hospital inpatients with somatic and psychiatric diseases. Clinical psychology \& psychotherapy, 8(3), 206-213.

Kinnear, P., R., \& Gray, C., D. (2010). IBM SPSS Statistics 18 Made Simple. New York: Psychology Press.

Labrecque, M., Rabhi, K., Laurin, C., Favreau, H., Moullec, G., Favreau, H., . . Julien, M. (2011). Can a self-management education program for patients with chronic obstructive pulmonary disease improve quality of life? Canadian Respiratory Journal, 18(5), e77-81.

Llewellyn, A. (2012). Understanding Quality of Life: Investigating the effects of individualised feedback on wellbeing. (PhD), University of Bath, Bath, UK.

Llewellyn, A., \& Skevington, S. M. (2014). The psychometric properties of measures used in interpreting quality of life: the WHOQOL-BREF, WHOQOL Importance and the GOSS-Q. Unpublished manuscript.

Marshall, S., Haywood, K. L., \& Fitzpatrick, R. (2006). Impact of patient-reported outcome measures on routine practice: a structured review. Journal of Evaluation in Clinical Practice, 12, 559-568. 
Mason, V. L., Skevington, S. M., \& Osborn, M. (2004). Development of a pain and discomfort module for use with the WHOQOL-100. Quality of Life Research, 13(6), 1139-1152.

Mills, M. E., Murray, L. J., Johnston, B. T., Cardwell, C., \& Donnelly, M. (2009). Does a patient-held quality-of-life diary benefit patients with inoperable lung cancer? Journal of Clinical Oncology, 27(1), 70-77.

Molzahn, A., Kalfoss, M., Skevington, S. M., \& Schick Makaroff, K. (2011). Comparing the importance of different aspects of quality of life to older adults across diverse cultures. Age and Ageing, 40(2), 192-199.

Molzahn, A., Skevington, S. M., Kalfoss, M., \& Schick Makaroff, K. (2010). The importance of facets of quality of life to older adults: an international investigation. Quality of Life Research, 19(2), 293-298.

National Institute for Health and Clinical Excellence. (2008). Guide to the methods of technology appraisal. London: NICE.

NHS England. (2013). Everyone counts: Planning for Patients 2013/2014. Retrieved 29th October, 2013, from http://www.england.nhs.uk/everyonecounts/

O'Carroll, R. E., Smith, K., Couston, M., Cossar, J. A., \& Hayes, P. C. (2000). A Comparison of the WHOQOL-100 and the WHOQOL-BREF in detecting change in quality of life following liver transplantation. Quality of Life Research, 9(1), 121-124.

Ostir, G. V., Smith, P. M., Smith, D., \& Ottenbacher, K. J. (2005). Reliability of the Positive and Negative Affect Schedule (PANAS) in medical rehabilitation. Clinical Rehabilitation, 19(7), 767-769.

Ovayolu, N., Ovayolu, O., \& Karadag, G. (2011). Health-related quality of life in ankylosing spondylitis, fibromyalgia syndrome, and rheumatoid arthritis: a comparison with a selected sample of healthy individuals. Clinical Rheumatology, 30(5), 655-664. 
Pickard, S. A., Wilke, C., Jung, E., Patel, S., Stavem, K., \& Lee, T. A. (2008). Use of a preference-based measure of health (EQ-5D) in COPD and asthma. Respiratory medicine, 102(4), 519-536.

Ramachandran, S., Lundy, J. J., \& Coons, S. J. (2008). Testing the measurement equivalence of paper and touch-screen versions of the EQ-5D visual analog scale (EQ VAS). Quality of Life Research, 17(8), 1117-1120.

Rosenbloom, S. K., Victorson, D. E., Hahn, E. A., Peterman, A. H., \& Cella, D. (2007). Assessment is not enough: A randomized controlled trial of the effects of HRQL assessment on quality of life and satisfaction in oncology clinical practice. PsychoOncology, 16(12), 1069-1079.

Salaffi, F., Carotti, M., Gasparini, S., Intorcia, M., \& Grassi, W. (2009). The health-related quality of life in rheumatoid arthritis, ankylosing spondylitis, and psoriatic arthritis: a comparison with a selected sample of healthy people. Health and Quality of Life Outcomes, 7.

Santana, M. J., Feeny, D., Johnson, J. A., McAlister, F. A., Kim, D., Weinkauf, J., \& Lien, D. C. (2010). Assessing the use of health-related quality of life measures in the routine clinical care of lung-transplant patients. Quality of Life Research, 19(3), 371-379.

Saxena, S., Carlson, D., Billington, R., \& Orley, J. (2001). The WHO Quality of Life Assessment Instrument (WHOQOL-Bref): The importance of its items for crosscultural research. Quality of Life Research, 10(8), 711-721.

Schwartz, L. A., \& Drotar, D. (2009). Health-Related Hindrance of Personal Goal Pursuit and Well-Being of Young Adults with Cystic Fibrosis, Pediatric Cancer Survivors, and Peers without a History of Chronic Illness. Journal of Pediatric Psychology, 34(9), 954-965. 
Skevington, S. M., \& Day, R. (2001). Developing a CD-ROM of the WHOQOL-BREF for use in primary care. Unpublished manuscript.

Skevington, S. M., Lotfy, M., \& O'Connell, K. A. (2004). The World Health Organization's WHOQOL-BREF quality of life assessment: Psychometric properties and results of the international field trial. A Report from the WHOQOL Group. Quality of Life Research, 13(2), 299-310.

Skevington, S. M., \& McCrate, F. M. (2012). Expecting a good quality of life in health: assessing people with diverse diseases and conditions using the WHOQOL-BREF. Health Expectations, 15(1), 49-62.

Skevington, S. M., \& O'Connell, K. A. (2004). Can we identify the poorest quality of life? Assessing the importance of quality of life using the WHOQOL-100. Quality of Life Research, 13(1), 23-34.

Slatkowsky-Christensen, B., Mowinckel, P., Loge, J. H., \& Kvien, T. K. (2007). Healthrelated quality of life in women with symptomatic hand osteoarthritis: A comparison with rheumatoid arthritis patients, healthy controls, and normative data. Arthritis \& Rheumatism, 57(8), 1404-1409.

Sniehotta, F. F. (2009). Towards a theory of intentional behaviour change: Plans, planning, and self-regulation. British Journal of Health Psychology, 14, 261-273.

Snyder, C. F., Blackford, A. L., Aaronson, N. K., Detmar, S. B., Carducci, M. A., Brundage, M. D., \& Wu, A. W. (2011). Can patient-reported outcome measures identify cancer patients' most bothersome issues? Journal of Clinical Oncology, 29(9), 1216-1220.

The EuroQoL Group. (2010). EQ-5D-5L. Retrieved 1st June, 2010, from http://www.euroqol.org/home.html 
The WHOQOL Group. (1995). The World Health Organization Quality Of Life Assessment (WHOQOL): Position paper from the World Health Organization. Social Science and Medicine, 41(10), 1403-1409.

The WHOQOL Group. (1998). Development of the World Health Organization WHOQOLBREF Quality of Life Assessment. Psychological medicine, 28(3), 551-558.

Valderas, J. M., Kotzeva, A., Espallargues, M., Guyatt, G., Ferrans, C. E., Halyard, M. Y., . . . Alonso, J. (2008). The impact of measuring patient-reported outcomes in clinical practice: A systematic review of the literature. Quality of Life Research, 17(2), 179193.

Velikova, G., Booth, L., Smith, A. B., Brown, P. M., Lynch, P., Brown, J. M., \& Selby, P. J. (2004). Measuring quality of life in routine oncology practice improves communication and patient well-being: a randomized controlled trial. Journal of Clinical Oncology, 22(4), 714-724.

Watson, D., Clark, L. A., \& Tellegen, A. (1988). Development and validation of brief measures of positive and negative affect: The PANAS scales. Journal of Personality and Social Psychology, 54(6), 1063-1070.

World Health Organization. (2010). ICD-10. 2nd. Retrieved 5th February, 2010, from http://www.who.int/classifications/icd/en/

Wu, C. (2009). Enhancing quality of life by shifting importance perception among life domains. Journal of happiness studies, 10(1), 37-47.

Yount, S. E., Rothrock, N., Bass, M., Beaumont, J. L., Pach, D., Lad, T., . . Del Ciello, K. (2013). A Randomized Trial of Weekly Symptom Telemonitoring in Advanced Lung Cancer. Journal of pain and symptom management, 47(6), 973-989. 
Table 1 Changes in QoL and comparison of administrative mode and health status groups (WHOQOL-BREF Domains and EQ-5D VAS MANOVA).

\begin{tabular}{|c|c|c|c|c|c|c|c|}
\hline \multicolumn{3}{|c|}{ Variable: } & $N$ & T1 & T1 & $\begin{array}{c}\mathrm{T} 2 \\
\text { mean }\end{array}$ & $\begin{array}{l}\text { T2 } \\
S D \\
\end{array}$ \\
\hline \multirow{16}{*}{ 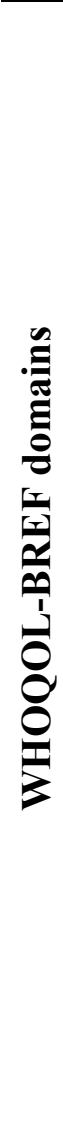 } & \multirow{4}{*}{ 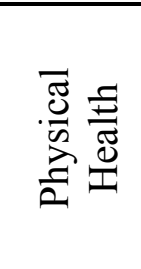 } & Ill & 79 & 63.78 & 19.15 & 64.94 & 18.81 \\
\hline & & Healthy & 42 & 79.54 & 16.07 & 81.46 & 14.95 \\
\hline & & Verbal guidance & 55 & 70.54 & 18.41 & 72.62 & 17.00 \\
\hline & & Written guidance & 66 & 68.17 & 20.58 & 69.06 & 20.85 \\
\hline & \multirow{4}{*}{ 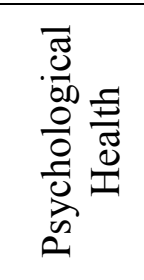 } & Ill & 79 & 63.44 & 15.27 & 65.61 & 15.99 \\
\hline & & Healthy & 42 & 67.64 & 11.95 & 70.08 & 11.20 \\
\hline & & Verbal guidance & 55 & 63.94 & 15.55 & 67.65 & 15.36 \\
\hline & & Written guidance & 66 & 65.69 & 13.23 & 66.76 & 14.08 \\
\hline & \multirow{4}{*}{ 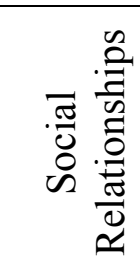 } & Ill & 79 & 68.35 & 18.21 & 69.36 & 19.18 \\
\hline & & Healthy & 42 & 69.84 & 19.73 & 72.52 & 20.93 \\
\hline & & Verbal guidance & 55 & 66.82 & 20.25 & 69.85 & 21.25 \\
\hline & & Written guidance & 66 & 70.58 & 17.23 & 70.96 & 18.60 \\
\hline & \multirow{4}{*}{ 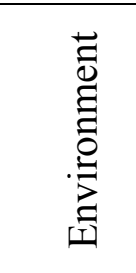 } & Ill & 79 & 74.25 & 13.25 & 75.59 & 13.47 \\
\hline & & Healthy & 42 & 78.79 & 12.89 & 79.47 & 12.96 \\
\hline & & Verbal guidance & 55 & 75.57 & 13.14 & 78.06 & 12.08 \\
\hline & & Written guidance & 66 & 76.04 & 13.44 & 76.01 & 14.39 \\
\hline \multirow{4}{*}{$\stackrel{0}{0}$} & & Ill & 79 & 68.80 & 17.40 & 69.14 & 17.22 \\
\hline & & Healthy & 42 & 82.64 & 11.94 & 82.12 & 12.98 \\
\hline & & Verbal guidance & 55 & 74.02 & 18.18 & 74.20 & 16.70 \\
\hline & & Written guidance & 66 & 73.26 & 16.10 & 73.18 & 17.35 \\
\hline \multicolumn{3}{|c|}{ MANOVA $d f=5,113$} & $F$ & $p$ & $\eta^{2}$ & & \\
\hline \multicolumn{3}{|c|}{ Within subjects effect of time } & 2.42 & $.040^{*}$ & .097 & & \\
\hline \multicolumn{8}{|c|}{ Between subjects' groups: } \\
\hline \multirow{2}{*}{\multicolumn{3}{|c|}{$\begin{array}{l}\text { Health status (ill / healthy) } \\
\text { Experimental condition (verbal / } \\
\text { written guidance) }\end{array}$}} & 6.16 & $.001^{* * *}$ & .214 & & \\
\hline & & & 0.42 & .836 & .018 & & \\
\hline \multicolumn{8}{|c|}{ Interactions: } \\
\hline \multicolumn{3}{|c|}{ Time*health status } & 0.33 & .896 & .014 & & \\
\hline \multicolumn{3}{|c|}{ Time*experimental condition } & 1.00 & .421 & .042 & & \\
\hline \multicolumn{3}{|c|}{$\begin{array}{l}\text { Time*health status } * \text { experimental } \\
\text { condition }\end{array}$} & 0.36 & .877 & .016 & & \\
\hline \multicolumn{3}{|c|}{ Health status*experimental cond'n } & 1.23 & .298 & .052 & & \\
\hline
\end{tabular}


Table 2 Differences in the importance of QoL in relation to health status groups, and differences between core QoL and Importance

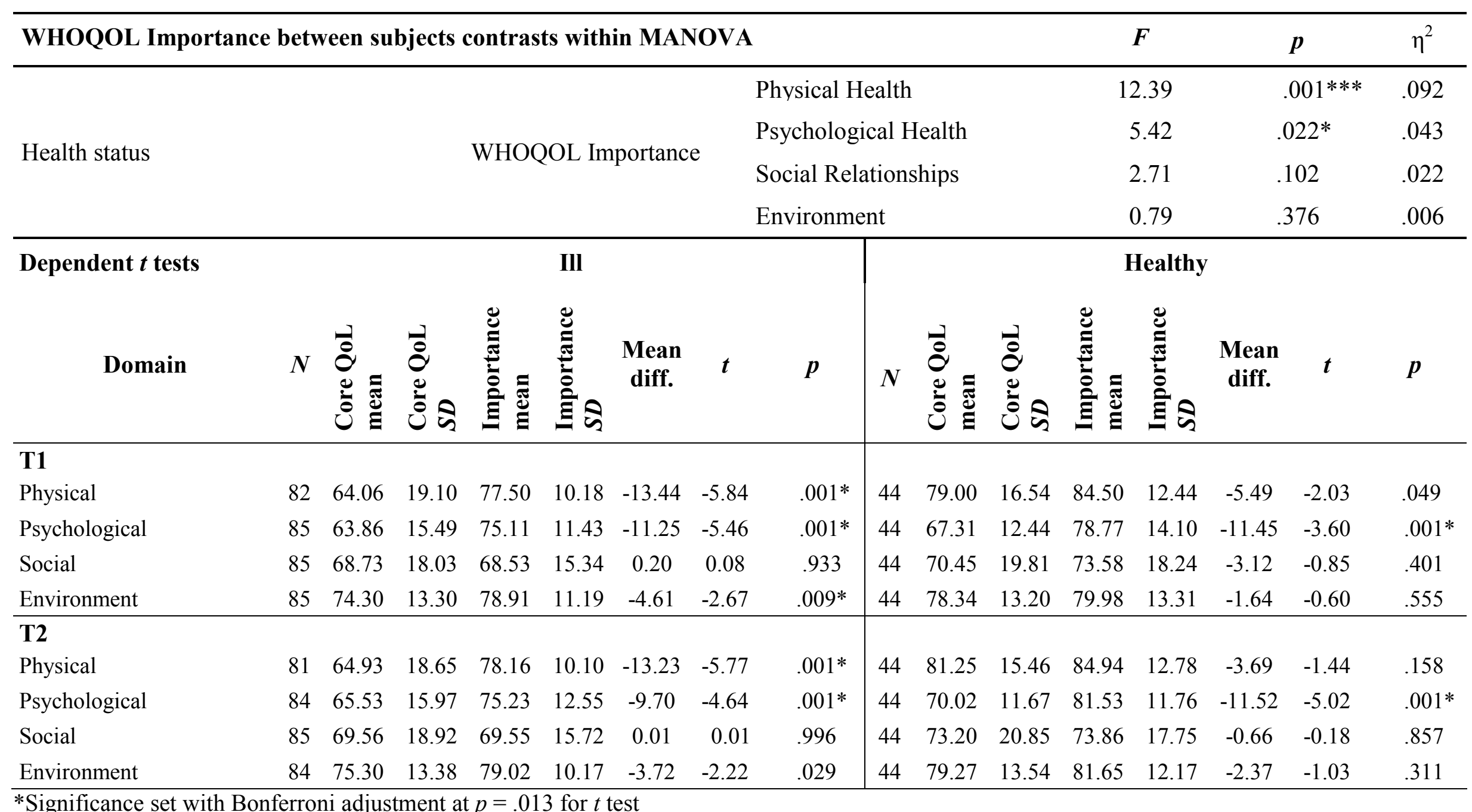


Figure 1 Example of a graphical summary profile of WHOQOL-BREF and Importance physical and psychological domain scores 